\title{
NBPF9 Gene May Be Involved in Congenital Hypopituitarism: A Whole-Genome Study of a Boy with Pituitary Stalk Interruption Syndrome and His Family
}

\author{
Cheng-Zhi Wang $\mathbb{D}^{1,2}$ Ling-Ling Guo, ${ }^{1,3}$ Qing-Hua Guo $\mathbb{D}^{1,4}$ and Yi-Ming Mu $\mathbb{D}^{1}$ \\ ${ }^{1}$ Department of Endocrinology, The First Medical Center of PLA General Hospital, Beijing 100853, China \\ ${ }^{2}$ Department of Endocrinology, Sun Yat-Sen Memorial Hospital, Sun Yat-Sen University, Guangzhou 510120, China \\ ${ }^{3}$ Department of Endocrinology, Beijing Electric Teaching Hospital of Capital Medical University, Beijing, 100073, China \\ ${ }^{4}$ Department of Endocrinology, Hainan Branch of Chinese PLA General Hospital, Sanya, Hainan 572000, China
}

Correspondence should be addressed to Qing-Hua Guo; gqh301@sina.com and Yi-Ming Mu; muyiming@301hospital.com.cn

Received 21 January 2020; Accepted 27 May 2020; Published 18 July 2020

Academic Editor: Maria L. Dufau

Copyright (c) 2020 Cheng-Zhi Wang et al. This is an open access article distributed under the Creative Commons Attribution License, which permits unrestricted use, distribution, and reproduction in any medium, provided the original work is properly cited.

\begin{abstract}
Pituitary stalk interruption syndrome (PSIS) is a rare congenital defect manifesting as various degrees of anterior pituitary hormone deficiency. Scattered familial cases have been found, revealing some genetic variants. However, most of the previous research studies involved an affected sibling, and the gene spectra of the patients' entire family have rarely been reported. We conducted a study of a family consisting of a PSIS patient with his unaffected sibling and healthy parents of Han Chinese background using whole-genome sequencing. Bioinformatic analysis was carried out, and mutations related to PSIS, singlenucleotide variants (SNVs), insertion-deletion (InDELs), and structural variations (SVs) in all the four samples were filtered. After Sanger sequencing, we confirmed the variants obtained and selected three candidate genes for functional verification. The gene variations in this boy with PSIS and his lineal relatives are reported herein; de novo sequencing revealed that the NBPF9 gene may be involved in the pathogenesis of PSIS.
\end{abstract}

\section{Introduction}

Pituitary stalk interruption syndrome (PSIS) is a rare congenital defect with an estimated incidence of $0.5 / 100,000$ births. PSIS causes various symptoms of pituitary hormone deficiency, including growth retardation and infertility [1-3].

Previous studies have revealed that genetic changes may contribute to its etiology. Studies on patients born to consanguineous parents have revealed germline mutations in some genes that may be responsible for the disease. For example, Fernandez-Rodriguez et al. reported a homozygous mutation, 301-302delAG, in the PROP1 gene in two consanguineous sisters [4]. Reynaud et al. identified a novel PROKR2 variant (p.Ala51Thr) in two familial cases [1]. In addition, Tatsi et al. [5] found a novel heterozygous nonsense mutation (c.799C > T, p.Q267X) in the TGIF gene in a female PSIS patient with a single central incisor; her father had the same mutation but was asymptomatic.

However, the above studies only provide partial details of the genetic variants responsible, as the samples were limited to siblings or one parent. In our study, we examined DNA from a PSIS boy, his unaffected parents, and his sister. We hope that our findings help in obtaining more information about this rare disease. Moreover, we performed functional verification of a novel gene found by sequencing to explain the pathogenesis in-depth.

\section{Materials and Methods}

2.1. Participants. A boy with PSIS was referred to Chinese PLA General Hospital; all his family members, including his 
unaffected sister and healthy parents, were also included. The study was approved by the Ethics Committee of the General Hospital of Chinese PLA, and informed consent was obtained from all participants prior to their participation in the study. The family lineage is displayed in Figure 1.

2.2. Whole-Genome Sequencing and Analysis. Genomic DNA was extracted from peripheral blood mononuclear cells (PBMCs) from the family members in our discovery cohort. Exome capturing was performed to collect whole exons of human genomic DNA. The exon-enriched DNA libraries were sequenced in $100 \mathrm{bp}$ paired-end reads using a HiSeq2000 sequencer (Illumina, San Diego, CA); coverage of 100x was typically achieved for each sample.

Qualified DNA was randomly fragmented using a Covaris Ultrasonic Processor. DNA libraries were constructed through end repair, addition of A to tails, purification, and PCR amplification. The pair-end libraries were sequenced using an Illumina HiSeq X Ten machine.

2.3. Genome Sequencing and Analysis. After verification, the different libraries were sequenced based on the effective concentration and required raw data by Illumina HiSeq X Ten sequencing platform after being verified. Clean reads were obtained after removing adaptor sequences and low mapping quality reads. Read alignment to the reference genome hg38 was performed with Burrows-Wheeler Aligner MEM (BWAMEM) (0.7.15). Duplicated reads were marked and removed by Picard (2.5.0). The alignment results were sorted using SAMtools (0.1.18). Recalibration of base quality scores and local realignment around InDELs were performed using Genome Analysis Toolkit (GATK) (3.6) [6]. SNVs and InDELs for each sample were determined using GATK HaplotypeCaller. Structural variants (SVs) were evaluated via LUMPY [7]. The database annotation, analysis, and global overview of variation were performed by ANNOVAR [8] and Variant Effect Predictor [9]. The genes SOX3, PROP1, LHX3, LHX4, HESX1, OTX2, PROKR2, TGIF, GPR161, and CDON have been analyzed in gene variation sets $[1,2,10-13]$.

2.4. Sanger Sequencing. Candidate variants were further confirmed by Sanger sequencing executed by Taihegene Biotechnology (Beijing). Purified PCR amplification products were obtained after amplification of target gene fragments in DNA samples using a Biometra PCR instrument. Chromas 2 software was used for manual interpretation, and sequences were obtained from NCBI GenBank (http://www. ncbi.nlm.nih.gov/sites/entrez/). The first-generation sequencing results were compared with the second-generation sequencing results, and genes were screened for functional verification. The primer sequences are shown in Table 1.

\subsection{Functional Verification}

2.5.1. Cell Culture and Transfection. Human embryonic stem cell (HESCS) H1 cells were cultured in mTeSR1 medium in pretreated culture plates covered with Matrigel (BD

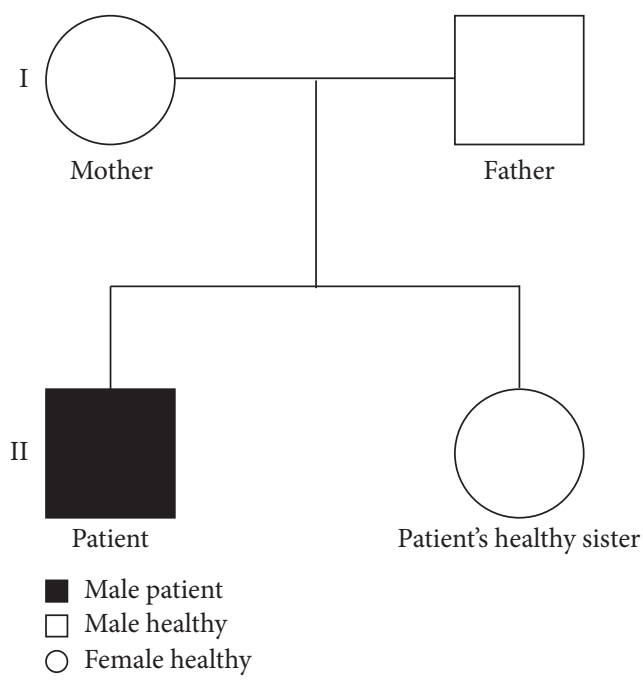

Figure 1: Pedigree of this family.

Table 1: Primer sequences.

\begin{tabular}{|c|c|}
\hline Gene & Primer sequence $\left(5^{\prime}-3^{\prime}\right)$ \\
\hline \multirow{2}{*}{ FCGBP } & F: $5^{\prime}$-AACgAggggTgAggTTCTTAC3' \\
\hline & R: $5^{\prime}$-CCACCACAAAACATCCTTTTCA- $3^{\prime \prime}$ \\
\hline \multirow{2}{*}{ RANGAP1 } & F: 5'-gAgACAACCAAAggAAgCCTgT-3' \\
\hline & R: 5'-CAgAgACATgAAggAggggAgT-3' \\
\hline \multirow{2}{*}{ FAM155A } & F: $5^{\prime}$-CAggCCTggAATTTgCTTTTAT-3' \\
\hline & R: 5'-gTTgCAgTAAggCAAgAgATCg-3' \\
\hline \multirow{2}{*}{ EPHA6 } & F: 5'-ATCTCAggACAgTTCCTATTC-3' \\
\hline & R: $5^{\prime}$-TTTTTCTgCAgCCCCggCTgTg-3' \\
\hline \multirow{2}{*}{ AGAP9 } & F: $5^{\prime}$-TgCTCTACCAAgTCTggTCTgC- $3^{\prime}$ \\
\hline & R: $5^{\prime}$-ATgTCATggAAAAAgTCgAgCA-3" \\
\hline \multirow{2}{*}{ NBPF9 } & F: $5^{\prime}$-CACTTgTACCCAggAgCCAgAg- $3^{\prime}$ \\
\hline & R: 5'-TggggACTAgACATCCCAgTCT-3' \\
\hline \multirow{2}{*}{ TYW1B } & F: $5^{\prime}$-gCTCTCCTTgTTgAATTgAT-3' \\
\hline & R: $5^{\prime}$-TgCATCAACTAATgggTgAAA- $3^{\prime}$ \\
\hline \multirow{2}{*}{ MUC12 } & F: 5'-ACCAAgCCTCAgTgAgAAATC-3' \\
\hline & R: 5'-ggACAgTgCTgTgTCAgTTg-3' \\
\hline
\end{tabular}

Company). The cells were passaged at approximately $70 \%$ confluence. siRNAs targeting MUC12, NBPF9, and TYW1B were designed by Gemma Company. Transfection was based on optimization methods reported in domestic and foreign literature [14-16]. For further experiments, RNA was extracted at $24 \mathrm{~h}$ after transfection and protein at $48 \mathrm{~h}$ after transfection.

2.5.2. Western Blotting. Protein samples were boiled ( $5 \mathrm{~min}$, $95^{\circ} \mathrm{C}$ ), separated by SDS-PAGE, and transferred onto polyvinylidene difluoride membranes. The membranes were blocked for $1 \mathrm{~h}$ in $5 \%$ bovine serum albumin (BSA) in TBST (20 mM Tris- $\mathrm{HCl}, \mathrm{pH} 7.6,150 \mathrm{mM} \mathrm{NaCl}$, and $0.1 \%$ Tween 20). Primary antibodies against LHX3 and PITX1 (Enogene Biotech, Nanjing, China) were diluted in TBST containing $5 \% \mathrm{BSA}$ and then applied to the membranes overnight at $4^{\circ} \mathrm{C}$ at a dilution of $1: 1,000$. After being washed three times with TBST, the membranes were incubated with horseradish peroxidase-conjugated secondary antibodies for $1 \mathrm{~h}$. The 
membranes were washed three times with TBST and developed with Chemiluminescence ECL Plus-Western Blotting detection reagents (Amersham Biosciences, Piscataway, NJ, USA).

\section{Results}

3.1. Clinical Features. The boy with PSIS was referred to our hospital due to growth retardation. He was born in a nonconsanguineous family, and none of his relatives had a history of growth retardation or short stature. He was born via breech delivery with a body weight of 3,200 $\mathrm{g}$ and a height of $50 \mathrm{~cm}$. After he was 5 years old, his parents noticed growth retardation compared with other children of his age. When he was then admitted to a local children's hospital, his height was $100 \mathrm{~cm}(-2.15$ SDS $)$, and he showed a delayed bone age of 3 . According to GH stimulation tests, the maximum value detected at the time was $0.69 \mathrm{ng} / \mathrm{mL}$, along with a low IGF-1 level estimated at $24.45 \mathrm{ng} / \mathrm{mL}$. Pituitary CT revealed a hypoplastic pituitary gland. He was diagnosed with GH deficiency, and hormone replacement therapy was initiated.

In 2013, when the boy was 8 years old, he was admitted to Chinese PLA General Hospital; his height was $106 \mathrm{~cm}(-1.07$ SDS), and he had a body weight of $16.5 \mathrm{~kg}$. He had a single central incisor, sharp palate, and single transverse palmar crease; he could not easily bend the fourth and fifth fingers of both hands. In addition to GH deficiency, he was diagnosed with hypothyroidism (free T4: $8.35 \mathrm{pmol} / \mathrm{mL}$ (reference interval: $12-22 \mathrm{pmol} / \mathrm{mL}$ ); TSH: $6.54 \mathrm{mU} / \mathrm{L}$ (reference interval: $0.35-5.5 \mathrm{mU} / \mathrm{L})$ ) and hypoadrenalism (F 8 am: $128.6 \mathrm{nmol} / \mathrm{L}$ (reference interval: $198.7-797.5 \mathrm{nmol} / \mathrm{L}$ at $24 \mathrm{~h}$ UFC) at admission. His bone age was that of a 5-year-old boy. The patient was treated with $\mathrm{GH}$, hydrocortisone, and levothyroxine thereafter. Records from the latest follow-up in 2015 showed a height of $131 \mathrm{~cm}(-1.06$ SDS $)$ and a bone age of approximately 8 years.

His younger sister, who was the second child in this family, exhibited normal growth with no deformities. As a 5year-old girl, her height was $111.5 \mathrm{~cm}$ and her weight was $18 \mathrm{~kg}$, which were in the normal range for her age. The results of her hormone laboratory tests were normal. The boy's parents denied consanguineous marriage or family histories of similar manifestations. The mother confirmed that she did not receive radiation or take any drugs during pregnancy. Figure 2 shows the appearance of the PSIS boy and his healthy sister.

\subsection{Molecular and In Silico Findings}

\subsubsection{Variant Statistics}

(1) SNVs and InDELs. We obtained 4,935,685 SNVs from the four samples as well as 9.644 InDELs; based on genetics, genomic region, and functional annotation, we filtered SNV results for each sample. $48.1 \%$ were intron variants and $37.7 \%$ were intergenic variants. In exon region, $52.3 \%$ were synonymous variants.

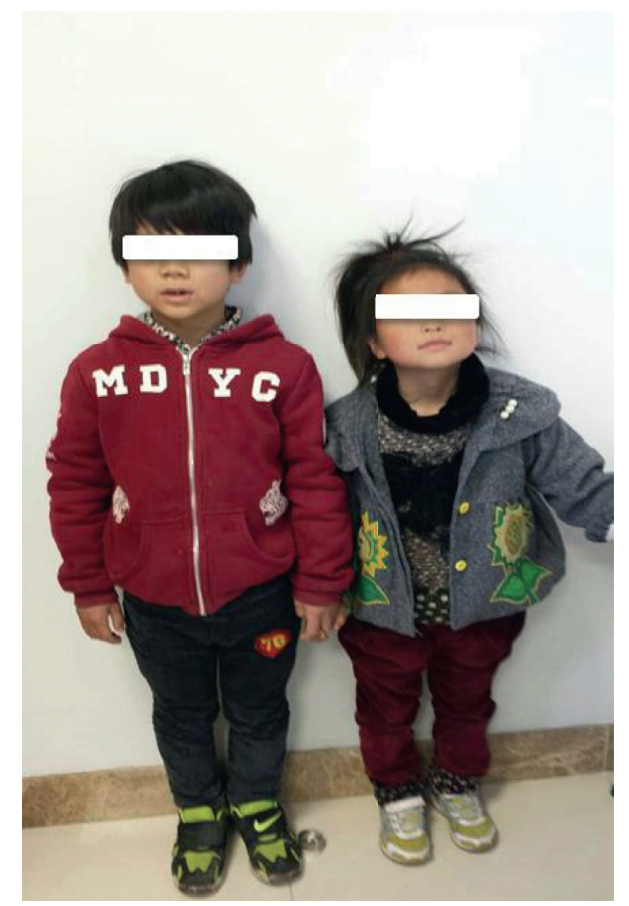

FIgure 2: The PSIS boy and his healthy sibling. The left is the PSIS boy ( 8 years old), and his healthy sister ( 5 years old) was on the right.

(2) Structural Variation (SV). LUMPY and the DGV database were used to assess all SVs in the 4 samples, SVs were detected in different regions of the genomic region, and 97.06\% SVs were located in intergenic region.

3.2.2. Analysis of Reported Genes. Mutations in the genes SOX3, PROP1, LHX3, LHX4, HESX1, OTX2, PROKR2, TGIF, GPR161, and CDON have been reported. The current database is not related to PSIS mutation locus information matching, according to the variation function for filtering exonic regions of the mutation loci. There are five genes that showed regional variation, including $L H X 4, P R O P 1, C D O N$, TGIF1, and PROKR2, as shown in Table 2.

3.3. De Novo Mutation Analysis. We aimed to find mutations that manifested as hybrid-type genome mutation loci in the PSIS boy but not genomic mutations in his parents. A total of 19 specific mutations were found (Table 3).

3.4. Inheritance Pattern Analysis. According to the pedigree information of the family, the variation and variation annotation information for each individual were used for grade selection; mutation loci were filtered by grade and function (nonsynonymous mutations) to obtain 267 candidate mutation loci. The 267 variation sites are located in 207 genes. Further analysis was performed by dbSNP, ClinVar, Exome Sequencing Project, 1000 Genomes Project, and Exome Aggregation Consortium database. Table 4 presents 11 filtered recessive genetic variations. 
Table 2: Analysis of reported genes.

\begin{tabular}{lccccccc}
\hline Pos & Gene & Chr & Start & End & Ref & Alt & Type \\
\hline Exonic & LHX4 & 1 & 180230592 & 180230592 & $\mathrm{~T}$ & $\mathrm{C}$ & Synonymous SNV \\
Exonic & LHX4 & 1 & 180274389 & 180274389 & $\mathrm{~A}$ & $\mathrm{G}$ & Nonsynonymous SNV \\
Exonic & PROP1 & 5 & 177995875 & 177995875 & $\mathrm{~T}$ & $\mathrm{C}$ & Nonsynonymous SNV \\
Exonic & PROP1 & 5 & 177995907 & 177995907 & $\mathrm{~A}$ & $\mathrm{G}$ & Synonymous SNV \\
Exonic & CDON & 11 & 125961075 & 125961075 & $\mathrm{~A}$ & $\mathrm{~T}$ & Nonsynonymous SNV \\
Exonic & CDON & 11 & 125978366 & 125978366 & $\mathrm{C}$ & $\mathrm{T}$ & Synonymous SNV \\
Exonic & CDON & 11 & 126021374 & 126021374 & $\mathrm{C}$ & $\mathrm{T}$ & Nonsynonymous SNV \\
Exonic & TGIF & 18 & 3457541 & 3457541 & $\mathrm{~A}$ & $\mathrm{G}$ & Synonymous SNV \\
Exonic & TGIF1 & 18 & 3457609 & 3457609 & $\mathrm{C}$ & $\mathrm{T}$ & Nonsynonymous SNV \\
Exonic & PROKR2 & 20 & 5302073 & 5302073 & $\mathrm{G}$ & $\mathrm{T}$ & Synonymous SNV \\
Exonic & PROKR2 & 20 & 5302085 & 5302085 & $\mathrm{G}$ & $\mathrm{A}$ & Synonymous SNV \\
Exonic & PROKR2 & 20 & 5302204 & 5302204 & $\mathrm{C}$ & $\mathrm{T}$ & Nonsynonymous SNV \\
Exonic & PROKR2 & 20 & 5302610 & 5302610 & $\mathrm{C}$ & $\mathrm{G}$ & Synonymous SNV \\
Exonic & PROKR2 & 20 & 5302730 & 5302730 & $\mathrm{G}$ & A & Synonymous SNV \\
\hline
\end{tabular}

TABle 3: Specific mutations in the PSIS boy.

\begin{tabular}{|c|c|c|c|c|c|c|c|}
\hline Pos & Gene & Chr & Start & End & Ref & Alt & Type \\
\hline Exonic & TRIOBP & chr22 & 37724147 & 37724147 & $\mathrm{G}$ & $\mathrm{A}$ & Nonsynonymous SNV \\
\hline Exonic & GP1BA & chr17 & 4933823 & 4933900 & AGC. ......CCC & - & Nonframeshift deletion \\
\hline Exonic & FCGBP & chr19 & 39902043 & 39902043 & $\mathrm{~A}$ & G & Nonsynonymous SNV \\
\hline Exonic & RANGAP1 & $\operatorname{chr} 22$ & 41254465 & 41254467 & TCC & - & Nonframeshift deletion \\
\hline Exonic & POTEG & chr14 & 19433854 & 19433854 & $\mathrm{C}$ & $\mathrm{T}$ & Nonsynonymous SNV \\
\hline Exonic & DNAJB6 & chr7 & 157385630 & 157385630 & G & $\mathrm{A}$ & Nonsynonymous SNV \\
\hline Exonic & FAM155A & $\operatorname{chr} 13$ & 107866364 & 107866365 & TG & - & Frameshift deletion \\
\hline Exonic & $\begin{array}{l}\text { FAM155A } \\
\text { GAGE2A, }\end{array}$ & chr13 & 107866367 & 107866379 & CGC.....GCT & - & Frameshift deletion \\
\hline Exonic & $\begin{array}{l}\text { GAGE2B, } \\
\text { GAGE2C }\end{array}$ & $\operatorname{chrx}$ & 49590729 & 49590729 & $\mathrm{C}$ & $\mathrm{T}$ & Nonsynonymous SNV \\
\hline Exonic & EPHA6 & chr3 & 96814795 & 96814797 & GAG & - & Nonframeshift deletion \\
\hline Exonic & FCGBP & chr19 & 39902109 & 39902109 & $\mathrm{~T}$ & G & Nonsynonymous SNV \\
\hline Exonic & TAL1 & chr1 & 47219924 & 47219925 & AG & - & Frameshift deletion \\
\hline Exonic & FLG & chr1 & 152312347 & 152312347 & $\mathrm{~A}$ & G & Nonsynonymous SNV \\
\hline Exonic & MUC12 & chr7 & 100997864 & 100997864 & G & $\mathrm{A}$ & Nonsynonymous SNV \\
\hline Exonic & RASA4B & chr7 & 102485138 & 102485138 & $\mathrm{C}$ & $\mathrm{T}$ & Nonsynonymous SNV \\
\hline Exonic & AGAP9 & chr10 & 47502991 & 47502991 & $\mathrm{C}$ & $\mathrm{T}$ & Nonsynonymous SNV \\
\hline Exonic & ZNF705E & chr11 & 71818808 & 71818808 & $\mathrm{~T}$ & $\mathrm{C}$ & Nonsynonymous SNV \\
\hline Exonic & NPIPB11 & chr16 & 29383805 & 29383805 & $\mathrm{C}$ & $\mathrm{T}$ & Nonsynonymous SNV \\
\hline Exonic & ZNF705E & chr11 & 71818809 & 71818809 & G & $\mathrm{A}$ & Stop-gain \\
\hline
\end{tabular}

TABle 4: Inheritance pattern analysis.

\begin{tabular}{|c|c|c|c|c|c|c|}
\hline Gene & Chr & Start & End & Ref & Alt & Type \\
\hline SPATA31A3 & $\operatorname{chr} 9$ & 66987043 & 66987043 & G & $\mathrm{T}$ & Nonsynonymous SNV \\
\hline OR9I1 & chr11 & 58118963 & 58118963 & G & $\mathrm{T}$ & Nonsynonymous SNV \\
\hline$A B C B 11$ & chr2 & 168944621 & 168944621 & G & $\mathrm{A}$ & Nonsynonymous SNV \\
\hline$A G A P 9$ & chr10 & 47503470 & 47503470 & G & $\mathrm{A}$ & Nonsynonymous SNV \\
\hline TYW1B & chr7 & 72728896 & 72728896 & $\mathrm{C}$ & $\mathrm{T}$ & Stop-gain \\
\hline$A G A P 9$ & chr10 & 47502579 & 47502579 & $\mathrm{C}$ & $\mathrm{T}$ & Nonsynonymous SNV \\
\hline$A G A P 9$ & chr10 & 47502650 & 47502650 & G & $\mathrm{C}$ & Nonsynonymous SNV \\
\hline NBPF9 & chr1 & 149075807 & 149075807 & $\mathrm{~A}$ & $\mathrm{C}$ & Nonsynonymous SNV \\
\hline$A G A P 9$ & $\operatorname{chr} 10$ & 47502343 & 47502343 & $\mathrm{~T}$ & $\mathrm{C}$ & Nonsynonymous SNV \\
\hline$A G A P 9$ & $\operatorname{chr} 10$ & 47502604 & 47502604 & $\mathrm{~A}$ & G & Nonsynonymous SNV \\
\hline ZNF180 & chr19 & 44497294 & 44497294 & A & G & Nonsynonymous SNV \\
\hline
\end{tabular}




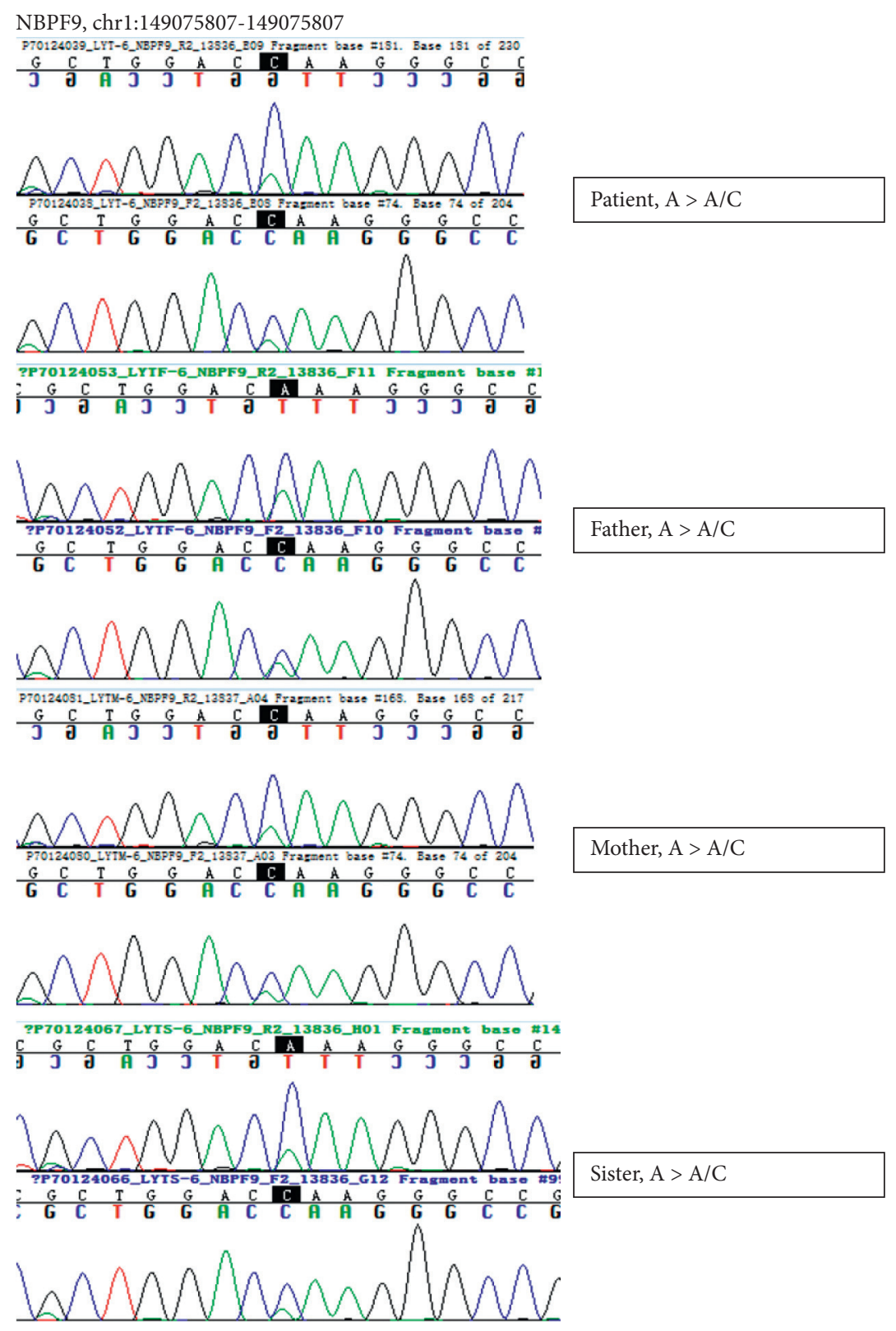

FIgURE 3: Mutations in the NBPF9 gene and Sanger sequencing validation.

As most of the above genes have rarely been studied, we selected NBPF9, MUC12, and TYW1B as candidate genes to further analyze their roles in nervous system development and cancer $[17,18]$.

3.5. Sanger Sequencing. We further confirmed the candidate variations by Sanger sequencing, and the results are depicted in Figure 3.

3.6. Protein Structure Prediction. After protein structure prediction, it was found that the NBPF9 p.L279W point mutation would cause protein structure variation. The conserved amino acid sequence is illustrated in Figure 4. This sequence shows good conservation.
3.7. Cell Culture and Western Blotting. Figure 5 shows the cell culture of HESCS (H1 cells). siRNAs targeting the candidate genes NBPF9, MUC12, and TYW1B were transfected into cells, and inhibition rates of more than $50 \%$ were obtained with the NBPF9 and MUC12 groups. At the protein level, LHX3 abundance decreased after transfection with NBPF9 siRNA. For PITX1, no significant change was observed between the NBPF9 transfection group and the control group.

\section{Discussion}

As a rare and complicated defect, PSIS is thought to be caused by genetic changes. However, genetic variants are found in only $5 \%$ of cases. Sporadic cases have been collected and studied, but little information was found. Through 


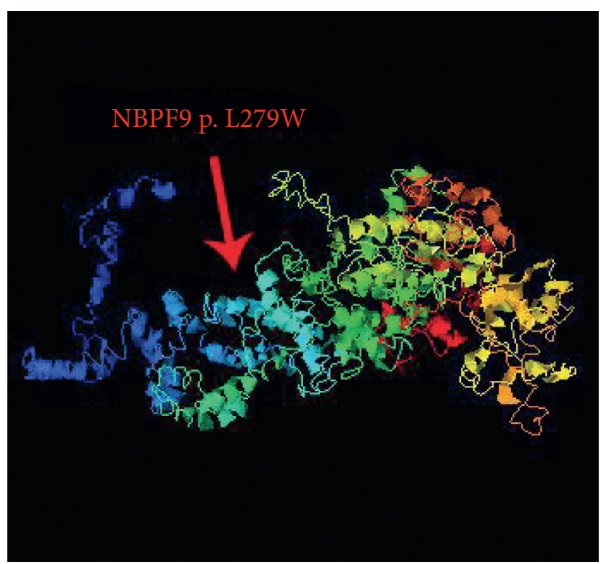

(a)

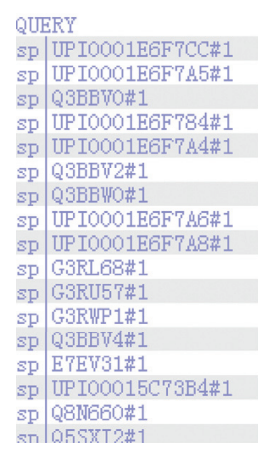

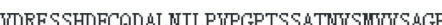

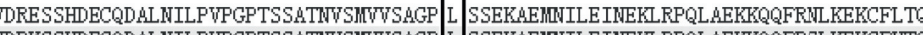
TDRKSSHDECQDALNILPVPGPTSSATNTSMWSAGP I SSEKAEMDILE INEKL RPQLAEKTRQFRSLKEKCFVTD WDRESSHDECQDALNILPVPGPTSSATNWSMWSAGP L SSEKMEMNILE INEKLRPQLAEKKQQFRSLKEKCFVTQ WDRESSHDECQDANNILPVPGPTSSATNWSMWSAGP L SSEKMEMDILEMREKLRPQLAEKKQQFRLLEKCFVTO WDRESSHDECQD \&LNILPVPGPTSSATNWSMWSAGP L SSEKAEMNILEINEKLRPQLAEKKQQFR SL KEKCFVTO DST TORE

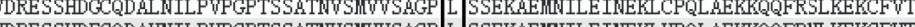
DRESSHDECQDANUILPVPGPTSSATNWSMWSAGP L SSEKAEMNILE INEKL HPQLAEKKQQFRULKEKCFVTO WDRESSHDECQDALNILPVPGPTSSATNTSMWSAGP L SSEKMEMNILEINEKLRPQLAEKKQQFRSLKEKCFVTO VDRESSHDECQDALINILPVPGPTSSATNTSMTVSAGP I SSEKAEMNILEINEKLRPQLAEKKQQFRSLKEKCFVTQ WDRESSHDECQDALIILPVPGPTSSATNWSMWSAGP I SSEKAEMNILEINEKLRPQLAEKKQQFRSLQEKCFVTQ TDRESSHDECQDALNILPVPGPTSSATNWSMWSAGP I SSEKAEMILILINEKLRPQLAEKKQQFRSLQEKCFVTO UDRESSHDECQDALNILPVPGPTSSATNWSMWSAGP L SSEKMEMDILE INEKLRPQLAEKKQQFRSLQEKCFVTO

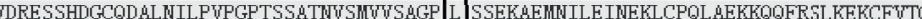

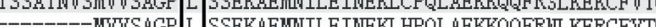
NLERCFVTQ MWWSAGP L SSEKAEMWILE INEKLRPQLAEKKQQRFRILKEKCFLTQ MWVSAGP L SSEKAEMLILEINEKLRPQLAEKKQQFRILKEKCFLTQ

(b)

FIGURE 4: Structure of the protein encoded by the NBPF9 gene. (a). Structure of the protein encoded by the NBPF9 gene, which would cause protein structure variation. (b). Conserved sequence in the region of the NBPF9 p.L279W mutation.

familial cases, we obtained some reported mutations, but in these studies, only the father or mother's DNA was examined. Here, we evaluate a family with a PSIS boy and a healthy sibling and parents, which may reveal more details of the etiology of PSIS

We analyzed the reported genes $L H X 4, P R O P 1, C D O N$, TGIF, and PROKR2 in our study. LHX4/LHX3 are members of the LIM-homeobox family and regulate the proliferation and differentiation of pituitary lineages $[1,2,12]$. PROP1 is involved in early patterning, proliferation, positional determination, and terminal differentiation of the pituitary gland $[4,13]$. CDON is a component of a cell-surface receptor complex that mediates cell-cell interactions between muscle precursor cells [11]. Tatsi reported TGIF as an active transcriptional corepressor of SMAD2 that may participate in the transmission of nuclear signals during development and in adults [5]. PROKR2 is thought to be involved in endocrine angiogenesis and neuronal migration and is expressed in the hypothalamus and pituitary gland [1]. However, none of the mutation frequencies were significant. In other words, the mutation frequencies were equal to those of the healthy group. We further performed inheritance pattern analysis and found 11 genes with related variations but that have rarely been studied; thus, little is known about their functions.

In our previous study related to sporadic cases, we proposed that compromised genetic interactions among multiple signaling pathways are likely to underlie the pathogenesis of sporadic PSIS [19]. Therefore, multigenetic causes may explain the pathogenesis of PSIS. Therefore, in this study, we attempted to identify novel gene variations. After bioinformatics analysis, we selected candidate variants for further research.

NBPF9 belongs to the neuroblastoma breakpoint family (NBPF) family, the members of which are characterized by binding to the repetitive DUF1220 protein domain. Gene copy number variation exists in the region of human chromosome 1q21. 1. DUF1220 domains have been associated with many diseases related to development and neurogenesis, such as small head deformity, autism and schizophrenia, mental retardation, congenital heart disease, neuroblastoma, and congenital kidney and urinary tract abnormalities, and previous studies have reported that DUF1220 is associated with lung cancer [17].

We found that the level of LHX3 decreased after transfection with NBPF9 siRNA. The LHX3 and PITX1 genes appear to play important roles during pituitary development. LHX3 is a LIM homologous domain transcription factor that is one of the earliest pituitary transcription factors expressed in development; it determines the specific differentiation of tissues and cells in vertebrates and invertebrates by regulating the expression of some genes. Currently, three types of LHX3 autosomal recessive inheritance have been identified in humans, and all patients have multiple pituitary hormone deficiencies [21-23]. PITX1, a member of 

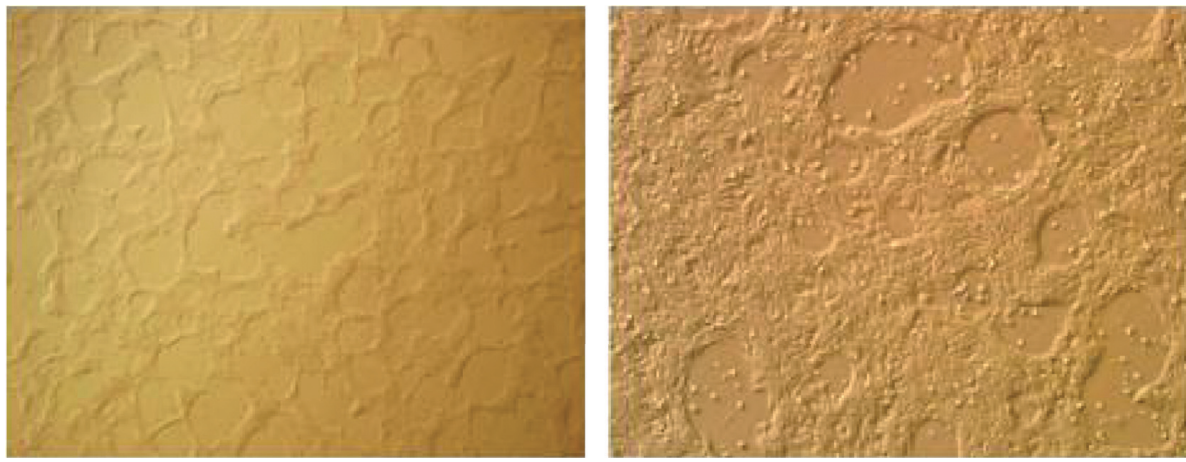

(a)

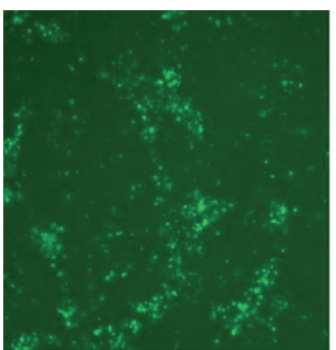

NBPF9 siRNA

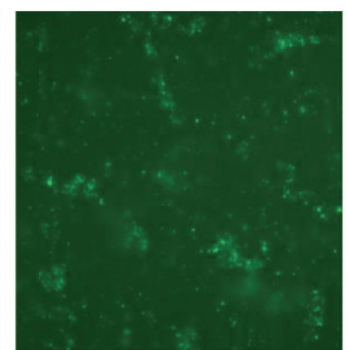

MUC12 siRNA

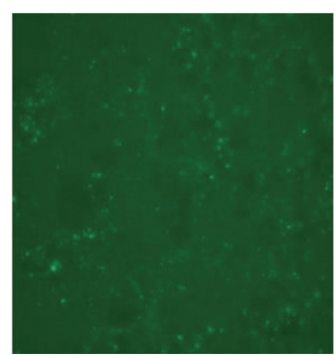

TYW1B siRNA

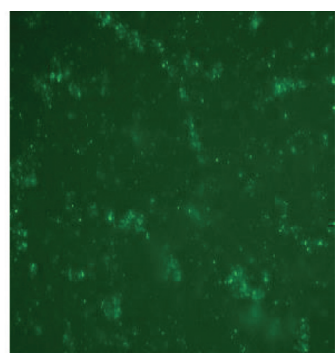

NC siRNA

(b)

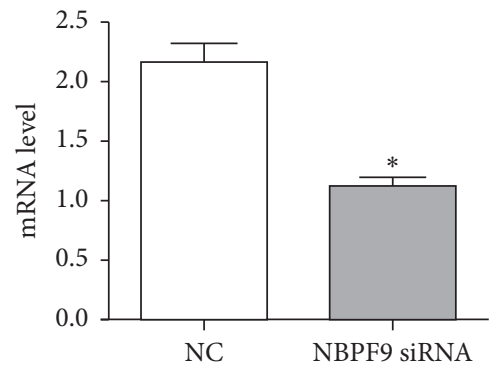

(a)

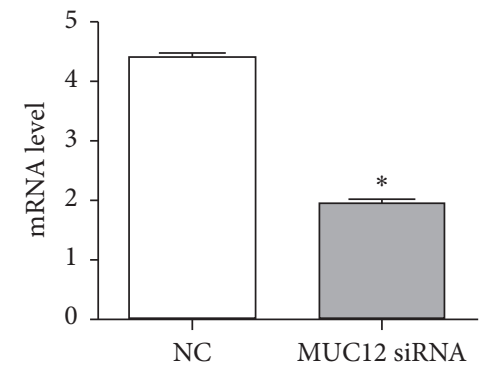

(b)

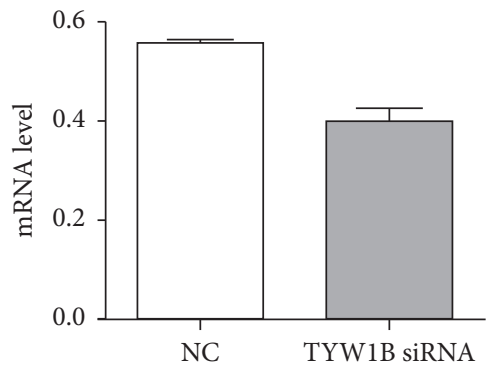

(c)

(c)

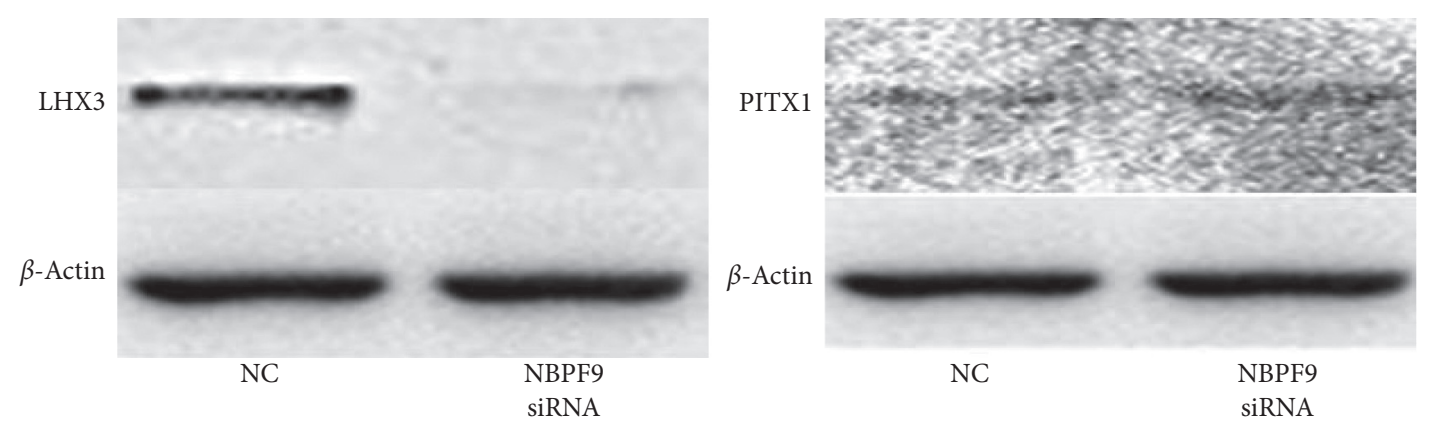

(d)

FIGURE 5: Culture status of human embryonic stem cells, siRNA transfection effect, and target gene expression. (a) Human embryonic stem cells on the left showed scattered growth and human embryonic stem cells on the right gradually formed cell colonies. (b) Cells after transfection under a fluorescence microscope. (c) siRNA transfection after $24 \mathrm{~h}$, with efficiency above $60 \%$. qPCR results after silencing the expression of each gene are shown below to verify the inhibitory effect. (d) Expression of LHX3 and PITX1 by western blotting, in which LHX3 was significantly decreased after treatment with siRNA targeting NBPF9.

the homologous domain protein transcription factor (PITX) family, was found in fruit flies in 1996; the encoded protein activates the transcription of opioid melanocortin precursor and is involved in the derivation and development of the pituitary gland [24]. The above research results indicate that LHX3 plays an important role in the development of the pituitary; in contrast, the NBPF9 gene has only been shown to be an important link in pituitary development. 
In our study, we describe the landscape of the gene spectrum of a PSIS family and, based on further experiments, speculate that the NBPF9 gene is likely a regulatory factor affecting the development of the pituitary. Nonetheless, more samples of entire PSIS families are needed to explain the exact pathogenesis.

\section{Conclusions}

The findings of this study provide considerable information about the gene spectrum of PSIS as a rare disease. However, there is a lack of evidence regarding all of the reported genes. More samples are needed to assess clinical applicability. The NBPF9 gene may be involved in congenital hypopituitarism [20].

\section{Abbreviations \\ PSIS: Pituitary stalk interruption syndrome \\ MRI: $\quad$ MR imaging \\ SNV: Single-nucleotide variants \\ InDEL: Insertion-deletion \\ SV: $\quad$ Structural variation \\ WES: Whole-exome sequencing \\ NGS: Next-generation sequencing.}

\section{Data Availability}

The data used to support the findings of this study are available from the corresponding author upon reasonable request.

\section{Conflicts of Interest}

The authors declare no conflicts of interest.

\section{Authors' Contributions}

Yi-Ming Mu and Qing-Hua Guo had designed the experiments; Cheng-Zhi Wang and Ling-Ling Guo accomplished the experiments; Cheng-Zhi Wang wrote the manuscript and revised it. Qing-Hua Guo and Yi-Ming Mu contributed equally to the work.

\section{Acknowledgments}

This work was supported by grants from (1) National Natural Science Foundation of China (Grant number: 81570705); (2) Key Research and Development Project of Hainan Province (Grant number: ZDYF2018117); (3) Sanya Science and Technology Innovation Project (Grant number: 2017YW16); (4) Hainan Provincial Health and Family Planning Commission Project (Grant number: 15A200071); (5) Yat-Sen Sailing Youth Fund (Grant number: YXQH201916); (6) Sun Yat-Sen Clinical Research Cultivating Program (Grant number: SYS-Q-202001). The authors greatly appreciate Prof. Ke Xing (Sun Yat-sen University, Guangzhou, China) for reviewing the manuscript and for helpful advice.

\section{References}

[1] R. Reynaud, S. A. Jayakody, C. Monnier et al., "PROKR2 variants in multiple hypopituitarism with pituitary stalk interruption," The Journal of Clinical Endocrinology \& Metabolism, vol. 97, no. 6, pp. E1068-E1073, 2012.

[2] R. Reynaud, M. Gueydan, A. Saveanu et al., "Genetic screening of combined pituitary hormone deficiency: experience in 195 patients," The Journal of Clinical Endocrinology \& Metabolism, vol. 91, no. 9, pp. 3329-3336, 2006.

[3] C.-Z. Wang, L.-L. Guo, B.-Y. Han, X. Su, Q.-H. Guo, and Y.-M. Mu, "Pituitary stalk interruption syndrome: from clinical findings to pathogenesis," Journal of Neuroendocrinology, vol. 29, no. 1, 2017.

[4] E. Fernandez-Rodriguez, C. Quinteiro, J. Barreiro et al., "Pituitary stalk dysgenesis-induced hypopituitarism in adult patients: prevalence, evolution of hormone dysfunction and genetic analysis," Neuroendocrinology, vol. 93, no. 3, pp. 181-188, 2011.

[5] C. Tatsi, A. Sertedaki, A. Voutetakis et al., "Pituitary stalk interruption syndrome and isolated pituitary hypoplasia may be caused by mutations in holoprosencephaly-related genes," The Journal of Clinical Endocrinology \& Metabolism, vol. 98, no. 4, pp. E779-E784, 2013.

[6] A. McKenna, M. Hanna, E. Banks et al., "The genome analysis toolkit: a mapreduce framework for analyzing next-generation DNA sequencing data," Genome Research, vol. 20, no. 9, pp. 1297-1303, 2010.

[7] R. M. Layer, C. Chiang, A. R. Quinlan, and I. M. Hall, "LUMPY: a probabilistic framework for structural variant discovery," Genome Biology, vol. 15, no. 6, p. R84, 2014.

[8] K. Wang, M. Li, and H. Hakonarson, "ANNOVAR: functional annotation of genetic variants from high-throughput sequencing data," Nucleic Acids Research, vol. 38, no. 16, p. e164, 2010.

[9] W. McLaren, L. Gil, S. E Hunt et al., "The ensembl variant effect predictor," Genome Biology, vol. 17, no. 1, p. 122, 2016.

[10] E. Karaca, R. Buyukkaya, D. Pehlivan et al., "Whole-exome sequencing identifies homozygousGPR161mutation in a family with pituitary stalk interruption syndrome," The Journal of Clinical Endocrinology \& Metabolism, vol. 100, no. 1, pp. E140-E147, 2015.

[11] A. Bashamboo, J. Bignon-Topalovic, H. Rouba et al., "A nonsense mutation in the hedgehog receptor CDON associated with pituitary stalk interruption syndrome," Journal of Clinical Endocrinology and Metabolism, vol. 101, no. 1, pp. 12-15, 2016.

[12] K. W. Sloop, E. C. Walvoord, A. D. Showalter, O. H. Pescovitz, and S. J. Rhodes, "Molecular analysis ofLHX3andPROP-1in pituitary hormone deficiency patients with posterior pituitary ectopia1," The Journal of Clinical Endocrinology \& Metabolism, vol. 85, no. 8, pp. 2701-2708, 2000.

[13] M. E. Melo, S. Marui, L. R. Carvalho et al., "Hormonal, pituitary magnetic resonance, LHX4 and HESX1 evaluation in patients with hypopituitarism and ectopic posterior pituitary lobe," Clinical Endocrinology, vol. 66, no. 1, pp. 95-102, 2007.

[14] H. Liu, C. Ren, B. Zhu et al., "High-efficient transfection of human embryonic stem cells by single-cell plating and starvation," Stem Cells and Development, vol. 25, no. 6, pp. 477-491, 2016.

[15] Y. Ma, J. Jin, C. Dong et al., "High-efficiency siRNA-based gene knockdown in human embryonic stem cells," RNA, vol. 16, no. 12, pp. 2564-2569, 2010.

[16] J. Yen, L. Yin, and J. Cheng, "Enhanced non-viral gene delivery to human embryonic stem cells via small molecule-mediated 
transient alteration of cell structure," Journal of Materials and Chemistry, vol. 2, no. 46, pp. 8098-8105, 2014.

[17] Y. Zhang, H. Wang, J. Wang et al., "Global analysis of chromosome 1 genes among patients with lung adenocarcinoma, squamous carcinoma, large-cell carcinoma, small-cell carcinoma, or non-cancer," Cancer and Metastasis Reviews, vol. 34, no. 2, pp. 249-264, 2015.

[18] J. K. Yamamoto-Furusho, I. Ascaño-Gutiérrez, J. FuruzawaCarballeda et al., "Differential expression of MUC12, MUC16, and MUC20 in patients with active and remission ulcerative colitis," Mediators of Inflammation, vol. 2015, Article ID 659018, 8 pages, 2015.

[19] T. Matsuyama, T. Ishikawa, K. Mogushi et al., "MUC12 mRNA expression is an independent marker of prognosis in stage II and stage III colorectal cancer," International Journal of Cancer, vol. 127, no. 10, pp. 2292-2299, 2010.

[20] Q.-H. Guo, C.-Z. Wang, Z.-Q. Wu et al., "Multi-genic pattern found in rare type of hypopituitarism: a whole-exome sequencing study of han chinese with pituitary stalk interruption syndrome," Journal of Cellular and Molecular Medicine, vol. 21, no. 12, pp. 3626-3632, 2017.

[21] M.-L. Sobrier, C. Brachet, M.-P. Vié-Luton et al., "Symptomatic heterozygotes and prenatal diagnoses in a nonconsanguineous family with syndromic combined pituitary hormone deficiency resulting from two novelLHX3 Mutations," The Journal of Clinical Endocrinology \& Metabolism, vol. 97, no. 3, pp. E503-E509, 2012.

[22] A. P. S. Bhangoo, C. S. Hunter, J. J. Savage et al., "A novelLHX3mutation presenting as combined pituitary hormonal deficiency," The Journal of Clinical Endocrinology \& Metabolism, vol. 91, no. 3, pp. 747-753, 2006.

[23] I. Netchine, M.-L. Sobrier, H. Krude et al., "Mutations in LHX3 result in a new syndrome revealed by combined pituitary hormone deficiency," Nature Genetics, vol. 25, no. 2, pp. 182-186, 2000.

[24] A. Esposito, A. M. Chiariello, M. Conte et al., "Higher-order chromosome structures investigated by polymer physics in cellular morphogenesis and differentiation," Journal of Molecular Biology, vol. 432, no. 3, pp. 701-711, 2019. 